University of Nebraska - Lincoln

DigitalCommons@University of Nebraska - Lincoln

Christian Binek Publications

Research Papers in Physics and Astronomy

November 2005

\title{
Reversible hysteresis loop tuning
}

\author{
Andreas Berger \\ Hitachi Global Storage Technologies, a.berger@nanogune.eu \\ Christian Binek \\ University of Nebraska-Lincoln, cbinek@unl.edu \\ D. T. Margulies \\ Hitachi Global Storage Technologies \\ A. Moser \\ Hitachi Global Storage Technologies \\ E. E. Fullerton \\ Hitachi Global Storage Technologies
}

Follow this and additional works at: https://digitalcommons.unl.edu/physicsbinek

Part of the Physics Commons

Berger, Andreas; Binek, Christian; Margulies, D. T.; Moser, A.; and Fullerton, E. E., "Reversible hysteresis loop tuning" (2005). Christian Binek Publications. 27.

https://digitalcommons.unl.edu/physicsbinek/27

This Article is brought to you for free and open access by the Research Papers in Physics and Astronomy at DigitalCommons@University of Nebraska - Lincoln. It has been accepted for inclusion in Christian Binek Publications by an authorized administrator of DigitalCommons@University of Nebraska - Lincoln. 


\title{
Reversible hysteresis loop tuning
}

\author{
A. Berger ${ }^{\mathrm{a}, *}$, Ch. Binek ${ }^{\mathrm{b}}$, D.T. Margulies ${ }^{\mathrm{a}}$, A. Moser $^{\mathrm{a}}$ and E.E. Fullerton ${ }^{\mathrm{a}}$ \\ a San Jose Research Center, Hitachi Global Storage Technologies, 650 Harry Road, San Jose, CA 95120 \\ ${ }^{b}$ Department of Physics and Astronomy, University of Nebraska-Lincoln, Lincoln, NE 68588
}

\begin{abstract}
We utilize antiferromagnetically coupled bilayer structures to magnetically tune hysteresis loop properties. Key element of this approach is the non-overlapping switching field distribution of the two magnetic layers that make up the system: a hard magnetic CoPtCrB layer (HL) and a soft magnetic CoCr layer (SL). Both layers are coupled antiferromagnetically through an only 0.6-nm-thick Ru interlayer. The nonoverlapping switching field distribution allows the measurement of magnetization reversal in the SL at low fields while keeping the magnetization state of the HL unperturbed. Applying an appropriate high field or high field sequence changes the magnetic state of the HL, which then influences the SL magnetization reversal due to the interlayer coupling. In this way, the position and shape of the SL hysteresis loop can be changed or tuned in a fully reversible and highly effective manner. Here, we study specifically how the SL hysteresis loop characteristics change as we move the HL through an entire high field hysteresis loop sequence.
\end{abstract}

Keywords: Hysteresis, Hysteresis tuning, AFC media, Magnetic thin films

\section{Introduction}

During the past century, magnetic materials have become a key building block of modern technology in a large number of application areas such as hard disk drives, power generators, motion sensors and credit cards to name a few. For all these diverse applications, magnetic properties have to be specifically tuned to very different optimal conditions. In particular, the requirements on magnetic hysteresis properties can vary greatly. Generator magnets, for instance, require minimal hysteresis, i.e. energy losses, whereas magnetic materials for disk media applications or hard magnets in general need to be strongly hysteretic to allow for stable information storage or stable operating conditions.

To fulfill such demands, magnetic materials have been tuned and optimized traditionally by using chemical and structural means like compound composition, segregation, shape, micro- or nano-structure (see, for instance [1]). These methods have been proven to be highly successful, given the wide-ranging technological applications of magnetic materials, but they have the drawback that once a material is prepared, its prop- erties are either fixed or require substantial structural or compositional changes to be altered again. It is, therefore, highly desirable to develop a methodology that allows the reversible alteration or fine tuning of magnetic properties, in particular the hysteresis behavior, which is independent from the particular chemical and crystallographic sample structure.

One example, where such an approach has already been successfully used in recent years is the application of the exchange bias effect (for a review on exchange bias, see for instance Ref. [2]). By coupling a ferromagnet to an antiferromagnet (AF), hysteresis loops have been found to shift their position depending on the field and temperature history [3]. This approach is nowadays utilized for biasing purposes in magnetic read sensors [4]. It allows for a certain flexibility in tuning magnetic hysteresis properties without micro-structural changes and is fully reversible as well. However, there are a number of drawbacks associated with this conventional exchange bias tuning. For instance, the setting of the exchange bias state requires field cooling, which makes a high temperature-processing step necessary for room temperature applications. Furthermore, antiferromagnetic materials are naturally inert to applied mag- 
netic fields, which severely limits the control of the magnetic state in the AF and the exchange bias itself. Also, AFs have no net moment, which makes it difficult to even characterize their magnetic state and typically does not allow a feedback control during the exchange bias setting process.

\section{Coupled ferromagnetic bilayer systems}

Recently, we demonstrated a novel pathway of tuning hysteresis loop properties in magnetic materials that is simple, very effective as well as fully reversible [5]. Following a strategy similar to the one used in exchange bias structures, we design our tunable magnetic material by combining two magnetic layers with very different properties [5]. Specifically, we use one hard ferromagnetic layer (HL) that will serve as the tuning element, hereby replacing the antiferromagnetic layer used in conventional exchange biased systems, and one soft ferromagnetic layer (SL) that is the actual tunable magnetic film. This bilayer structure has the advantage that the pre-set tuning field and temperature ranges, which are necessary to bring the HL into the desired pre-conditioned state, are much more accessible than in the case of traditional exchange bias structures using AFs. For the functionality of this approach, it is necessary that both layers be coupled to achieve tunability of the SL magnetization reversal. This coupling has to be strong enough to produce a considerable effect, but cannot be so strong that the individual character of each layer vanishes. Such an intermediate coupling strength can be achieved by utilizing the AFinterlayer exchange coupling [6] and [7].

The structures that were successfully utilized in our novel hysteresis tuning approach consist of a 15-nm-thick hardmagnetic CoPtCrB film, the tuning layer HL, exchange coupled by means of an only 6 - $\AA$-thick Ru-interlayer to a 1-2-nm-thick CoCr film, which is the tunable layer SL. Similar AF-coupled bilayer structures were recently introduced as disk recording media due to their superior stability and performance characteristics [8]. A schematic of this structure can be seen in Fig. 1, which also displays the experimentally measured hysteresis loop behavior. The thin solid line shows the major hysteresis loop, which exhibits two clearly distinguishable magnetization reversal steps, one for the SL at low fields and one for the $\mathrm{HL}$ at approximately $\pm 4 \mathrm{kOe}$. The two curves shown as thick solid lines are two separately measured minor loops, after coming from positive and negative saturation, respectively. These curves show the reversal of the SL alone. The hysteretic portions of these loops are shifted from the origin by a bias field $h$ bias due to the AF coupling to the positively or negatively magnetized $\mathrm{HL}$, which has a remanent magnetization $\mathrm{Mr}$. Both curves are closed and merge well with the respective part of the major hysteresis curve, which verifies that the two layers have clearly separated switching field distributions as previously demonstrated [5]. Thus, it is possible in a first step to set the HL magnetization at high magnetic fields and then measure the hysteresis properties of the SL at low fields without perturbing the HL magnetization state.

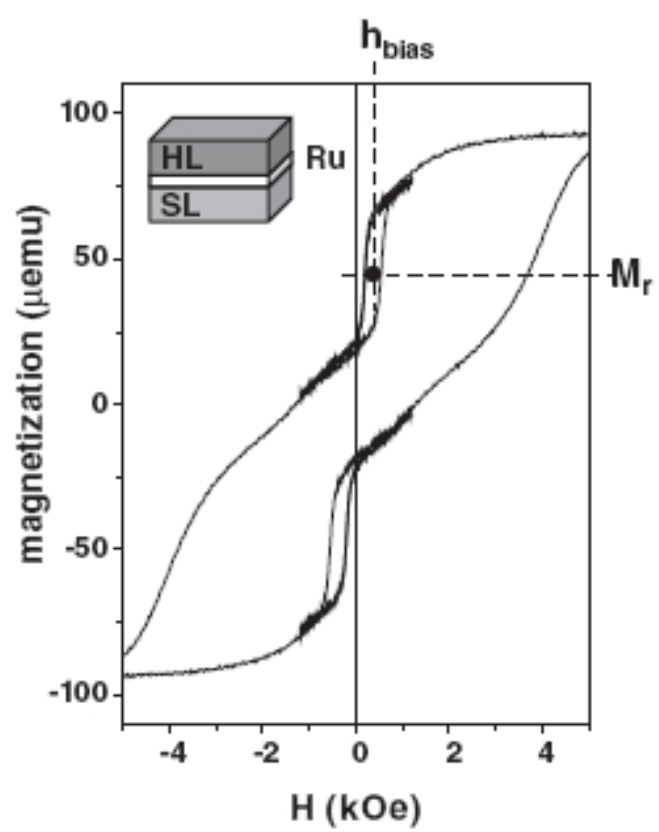

Fig. 1. Experimental magnetization vs. applied field curves, measured for an AF-coupled bilayer structure consisting of a 15-nm-thick CoPtCrB-alloy hard layer (HL) and a $1.5 \mathrm{~nm} \mathrm{CoCr-film}$ as the soft layer (SL). Both are separated by a $6 \AA \mathrm{Ru}$-film. The thin solid line is the major loop, showing the well-separated reversal of both layers. The thick solid lines are low field minor loops after positive and negative saturation, respectively, which are dominated by the magnetization reversal of the SL alone. The SL hysteresis loops are shifted from the origin due to the remanent magnetization $\mathrm{M}_{\mathrm{r}}$ of the $\mathrm{HL}$ and the Rumediated interlayer exchange bias $h_{\text {bias }}$. The inset is a schematic of the sample structure.

The detailed procedure used for tuning purposes in this study is shown in Fig. 2, which displays the field vs. time sequence applied to the sample. Fig. 2a shows that the entire sample is at first demagnetized once by applying an alternating field of decreasing amplitude in step I. The applied starting field of this sequence is $10 \mathrm{kOe}$. Subsequently, the field amplitude is reduced by $0.1 \%$ per cycle. This AC-demagnetization procedure produces states with magnetization values of consistently less than $5 \%$ of the sample's remanent magnetization after saturation. Subsequently, the sample is partially re-magnetized in step II by applying a certain set field $\mathrm{H}_{\text {set }}$ in the range of up to $10 \mathrm{kOe}$. After these two preconditioning steps I and II, the low field hysteresis loop of the SL is measured in step III with a maximum field of $\mathrm{H} m= \pm 1.2 \mathrm{kOe}$. Steps II and III are then repeated numerous times for different values of Hset producing different magnetization states in the HL, which in turn alter the magnetization reversal behavior of the adjacent SL. In this study, the $\mathrm{H}_{\text {set }}$ sequence is chosen to follow an entire hysteresis loop of the HL, including the virgin curve as schematically shown in Fig. 2b. At each point on this Hset curve, a minor loop of the SL magnetization reversal is measured and analyzed.

\section{Hystersis tuning results}

How the various properties of the bilayer structure are affected by the above described field sequence, can be seen in 
(a)
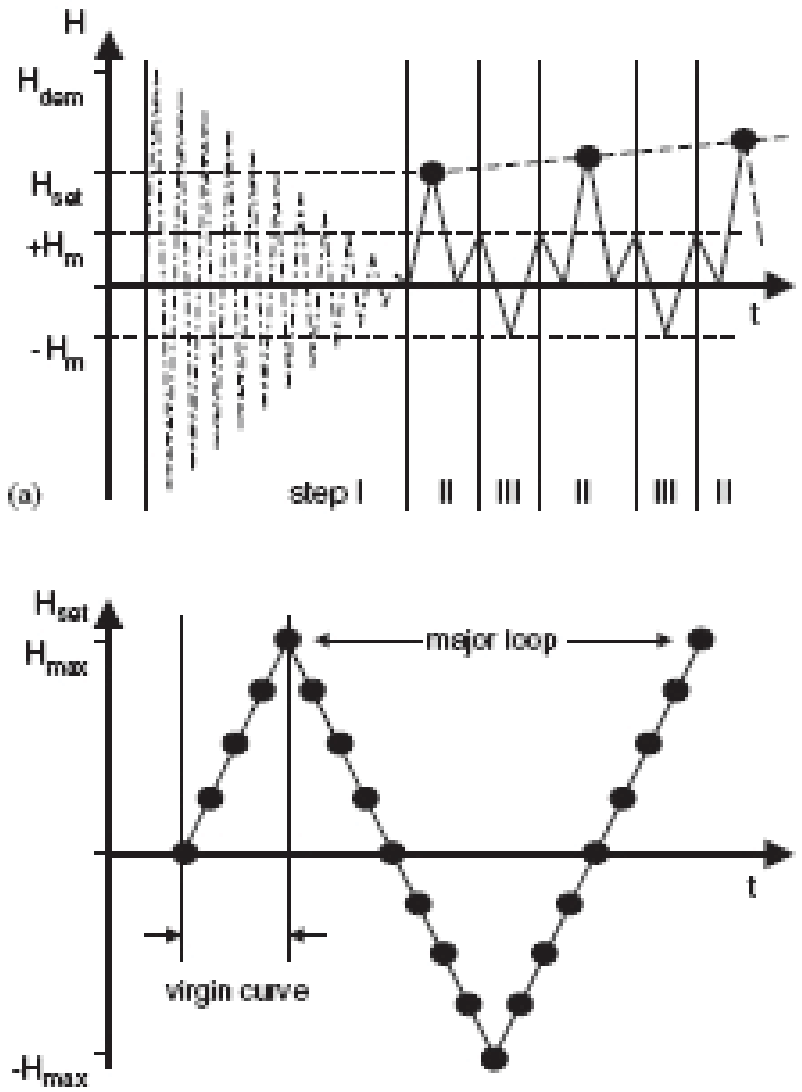

(b)

Fig. 2. Applied magnetic field $\mathrm{H}$ vs. time $\mathrm{t}$ sequence of the measurement procedure: (a) the entire sample is AC-demagnetized in step I, starting with a maximum applied field of $\mathrm{H}_{\mathrm{dem}}=10 \mathrm{kOe}$. In step II, the HL is partially re-magnetized by applying a variable field $\mathrm{H}_{\mathrm{set}}$, and in step III the low field response of the SL is measured (thick solid line) for fields $\mathrm{H}_{\mathrm{mo}}<\mathrm{H}<+\mathrm{H}_{\mathrm{m}}$. Steps II and III are then repeated multiple times for different values of Hset; (b) time evolution of Hset for the complete experiment $\left(\mathrm{H}_{\max }=10 \mathrm{kOe}\right)$.

Fig. 3. Fig. 3a shows the remanent magnetization $\mathrm{Mr}$ of the $\mathrm{HL}$ as a function of $\mathrm{H}_{\text {set }} \cdot \mathrm{M}_{\mathrm{r}}$ is determined from the magnetization offset of the low field minor loop as shown in Fig. 1.The different symbols correspond to the three different parts of the $\mathrm{H}_{\text {set }}$ sequence. The open squares $(\square)$ were measured first with $\mathrm{H}_{\text {set }}$ increasing from 0 to $\mathrm{H}_{\max }=+10 \mathrm{kOe}$ after the entire sample was AC demagnetized once by the step I sequence shown in Fig. 2a.In the second part of the measurement shown as solid circles $(\bullet)$, the set field was stepwise lowered from +10 to -10 kOe. Afterwards, Hset was stepwise increased from -10 to +10 kOe with the corresponding data shown as solid triangles $(\boldsymbol{\Lambda})$. Fig. $3 b$ shows the exchange bias field hbias acting upon the SL. This curve it virtually indistinguishable from the $\mathrm{M}_{\mathrm{r}}\left(\mathrm{H}_{\text {set }}\right)$ curve, which is consistent with our previous observation that the sample-averaged SL exchange bias is almost exactly proportional to the HL magnetization [5] and [9]. The linear correlation coefficient between the $h_{\text {bias }}{ }^{-}$and $\mathrm{M}_{\mathrm{r}}$-data sets of Fig. 3 is $R=0.9983$, verifying the proportionality, hbias $\boldsymbol{\Delta} \sim \mathrm{M}_{\mathrm{r}}$, for a complete hysteresis loop cycle of the HL. This finding is a more general observation than the results of our previous stud-
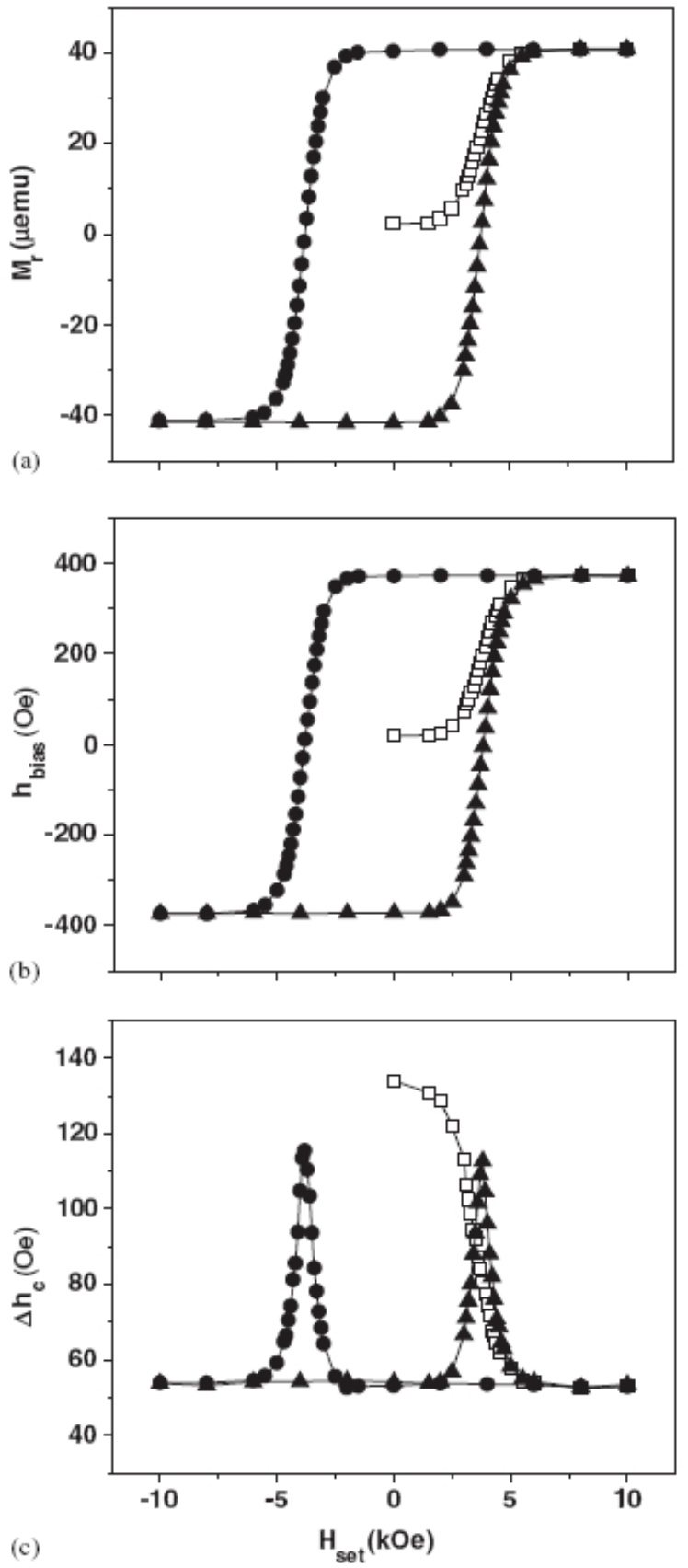

Fig. 3. Experimental data for various magnetic properties as a function of $\mathrm{H}_{\text {set? }}$ ? measured for the same AF-coupled bilayer structure shown in Fig. 1 and following the procedure given in Fig. 2: (a) remanent magnetization $M_{r}$ of the $\mathrm{HL}$, determined from the magnetization axis minor loop shift; (b) exchange bias field hbias, determined from the field axis minor loop shift; (c) SL magnetization reversal width $\Delta h_{\mathrm{c}}$ [10], determined from the slope of the minor loops. The different symbols correspond to $(\square)$ the virgin curve after demagnetization, ( $\bullet$ ) the curve for decreasing $\mathrm{H}_{\text {set }}$ and $(\boldsymbol{\Delta})$ the curve for increasing $\mathrm{H}_{\text {set }}$.

ies, because those were limited to the virgin curve segment of the HL hysteresis loop only [5] and [9].

The rather simple relationship between hbias and $M_{r}$ is due to the fact that the hard magnetic material is itself a rather thin film, which does not allow for any significant three-dimensional magnetization structure normal to the interface. Therefore, 
the volume averaged magnetization $\mathrm{M}_{\mathrm{r}}$ is identical to the interface magnetization in the HL, which is responsible for the interlayer coupling and the exchange bias shown by the SL.

Fig. 3c shows how $\Delta h \mathrm{C}$, the width of the SL magnetization reversal region [10], evolves as the HL is tuned through its hysteresis loop. As observed previously, we find that $\Delta h \mathrm{C}$ is rather large for the demagnetized HL state, which corresponds to a disordered state of locally applied bias fields. As the HL is magnetized, this disorder is reduced and $\Delta h \mathrm{C}$ gets smaller. Recently, we demonstrated that the reduction of $\Delta h \mathrm{C}$ upon magnetizing the HL has actually two components: the reduced distribution width of the locally applied bias fields, and the increase of the magnetization reversal correlation length [11]. The increase in correlation length is hereby due to the intergranular exchange coupling within the SL, which tends to synchronize the magnetization reversal against the influence of the locally varying bias fields [11]. Overall the SL magnetization reversal is very much similar to results from theoretical models like the Random Field Ising Model (RFIM), for instance, in which each of the coupled magnetic units is exposed to a locally varying bias field. Calculations of magnetic hysteresis properties for the RFIM find a strong dependence of the collective reversal behavior from the distribution width $\Delta h_{\text {bias }}$ of the locally varying fields [12]. In particular, it has been found theoretically that $\Delta h \mathrm{C}$ decreases with decreasing $\Delta h_{\text {bias }}$ as it does in our measurements. Additionally, a non-equilibrium phase transition of the reversal process can occur for a critical distribution width, at which the reversal mode switches from a local to a completely collective phenomenon [12]. Such disorder-tuned transitions of the reversal behavior have also been observed experimentally [13] and [14].

The $\Delta h \mathrm{C}$-data, shown in Fig. $3 \mathrm{c}$, are characterized by two rather sharp peaks that coincide with the zero-crossings of $\mathrm{M}_{\mathrm{r}}$ along the HL major loop. This is the expected result, because at or near the DC-demagnetized state, the magnetic order inside the $\mathrm{HL}$ is at its lowest point during the major loop reversal process and, therefore, the bias field distribution, acting upon the SL, is the widest. This, in turn, widens the magnetization reversal region of the SL hysteresis loop. These two peaks of $\Delta \mathrm{hC}$ are comparable in their width and amplitude with each other, but they both are considerably lower in their peak value than the demagnetized starting point of the measurement. This result reflects the fact that DC-demagnetized states in general are more correlated than an AC-demagnetized state that is the result of many hysteresis loops with decreasing amplitude. Correspondingly, the SL bias field distribution width due to such a DC-magnetized HL is narrower than in the AC-demagnetized case.

Fig. 4 shows the hysteresis loop losses for the SL minor loops as a function of $\mathrm{H}_{\text {set }}$. We observe that the losses are the largest for the DC-demagnetized states, while they are the lowest for the well-ordered HL magnetization states. This is not an unusual observation for materials in general, but it would be interesting to compare this finding to the behavior of the RFIM. To our knowledge, the effect of disorder on the hystere- sis loop loss has not yet been studied in such models. A similar effect, however, has been previously observed in a traditional exchange bias system, for which an $\mathrm{HC}$ increase was reported upon suppressing the net exchange bias [15]. The data in Fig. 4 also show that the SL hysteresis losses for the DC-demagnetized HL states are higher than the loss for the AC-demagnetized state. This appears to be somewhat surprising, because it is quite different from the previously discussed $\Delta \mathrm{hC}$ vs. Hset behavior (Fig. 3c), for which we found the largest disorder effect in the AC-demagnetized state. It would indeed appear to be a contradiction if our bilayers were made up from simple Ising spins. However, the SL grains in our samples have only a rather small and locally varying magneto-crystalline anisotropy. Thus, the SL can accommodate disordered exchange bias by more complex non-collinear magnetization structures. Such complex structures were recently observed in AF-coupled bilayer samples and found to be responsible for the occurrence of bi-quadratic interlayer exchange coupling [16]. Therefore, the exchange bias disorder level for the AC-demagnetized HL might be large enough to cause a partial reorientation of the local SL magnetization states in remanence. In such a scenario, one would expect the SL hysteresis loops to exhibit large $\Delta h \mathrm{C}$ values as well as an only modest hysteresis loss similar to our experimental findings here.

In conclusion, we demonstrate a novel methodology, which allows the tuning of hysteresis properties for magnetic materials. In contrast to conventionally available methods that are based upon irreversible changes in materials chemistry and micro-structure, our new method is based on the magnetic presetting of an adjacent hard magnetic film, which is fully reversible and allows for a simple, accurate and very effective tuning.

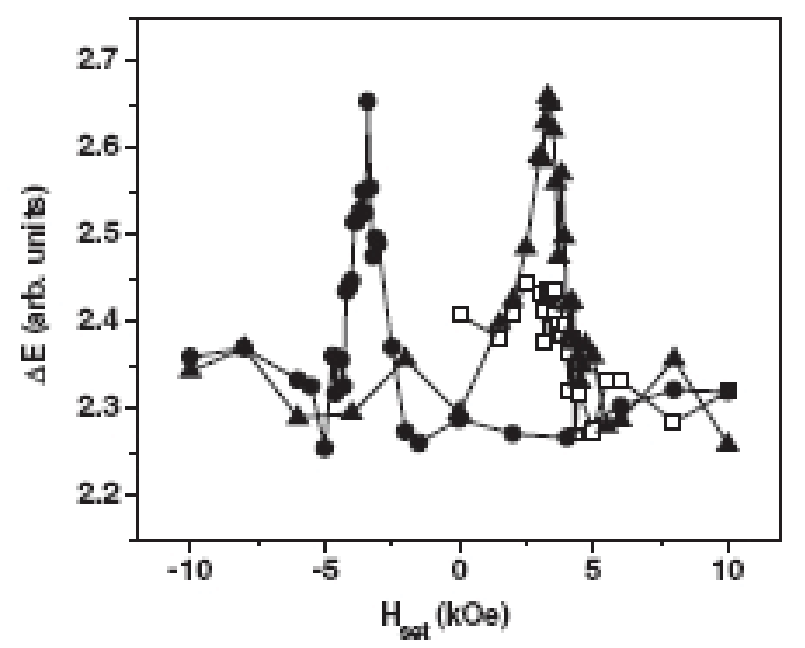

Fig. 4. Energy loss $\Delta \mathrm{E}$ per completed minor loop as a function of $\mathrm{H}_{\text {set }}$, measured for the same AF-coupled bilayer structure shown in Fig. 1 and following the procedure given in Fig. 2. The different symbols correspond to ( $\square$ ) the virgin curve after demagnetization, $(\bullet)$ the curve for decreasing $\mathrm{H}_{\text {set }}$, and $(\boldsymbol{\Delta})$ the curve for increasing $\mathrm{H}_{\text {set }}$. 


\section{Acknowledgements}

Work at the University of Nebraska was supported by the MRSEC Program of the NSF (DMR-0213808).

\section{References}

[1] C.-W. Chen, Magnetism and Metallurgy of Soft Magnetic Materials, North Holland Publishers, Amsterdam (1977).

[2] A.E. Berkowitz and K. Takano, J. Magn. Magn. Mater. 200 (1999), p. 552.

[3] W. Meiklejohn and C.P. Bean, Phys. Rev. 102 (1956), p. 1413.

[4] B. Dieny et al., J. Appl. Phys. 69 (1992), p. 4774.

[5] A. Berger, D.T. Margulies and H. Do, Appl. Phys. Lett. 85 (2004), p. 1571.

[6] P. Grünberg, R. Schreiber, Y. Pang, M.B. Brodsky and H. Sowers, Phys. Rev. Lett. 57 (1986), p. 2442.

[7] S.S.P. Parkin, N. More and K.P. Roche, Phys. Rev. Lett. 64 (1990), p. 2304.
[8] Eric E. Fullerton, D.T. Margulies, N. Supper, H. Do, M. Schabes, A. Berger and A. Moser, IEEE Trans. Magn. 39 (2003), p. 639.

[9] A. Berger, D.T. Margulies and H. Do, J. Appl. Phys. 95 (2004), p. 6660.

[10] $\triangle \mathrm{hC}$ is determined by fitting the expression $\mathrm{M}(\mathrm{h})=$ MStan-1 $[(\mathrm{h}-\mathrm{hC}) / \Delta \mathrm{h}]$ to individual sets of magnetization reversal data. Here, MS and hC are the saturation magnetization and the coercivity of the SL, respectively.

[11] A. Berger, D.T. Margulies, H. Do, A. Ktena and K. Dahmen, J. Appl. Phys. 97 (2005), p. 10K109.

[12] J.P. Sethna et al., Phys. Rev. Lett. 70 (1993), p. 3347.

[13] A. Berger, A. Inomata, J.S. Jiang, J.E. Pearson and S.D. Bader, Phys. Rev. Lett. 85 (2000), p. 4176.

[14] J. Marcos et al., Phys. Rev. B 67 (2003), p. 224406.

[15] C. Leighton, J. Nogues, B.J. Jonsson-Akerman and I.K. Schuller, Phys. Rev. Lett. 84 (2000), p. 3466.

[16] A. Moser, A. Berger, D.T. Margulies and E.E. Fullerton, Phys. Rev. Lett. 91 (2003), p. 097203. 